\title{
Auditing Search Engines for Differential Satisfaction Across Demographics
}

\author{
Rishabh Mehrotra* \\ r.mehrotra@cs.ucl.ac.uk \\ University College London \\ Amit Sharma \\ amshar@microsoft.com \\ Microsoft Research
}

\author{
Ashton Anderson \\ ashton@microsoft.com \\ Microsoft Research \\ Hanna Wallach \\ wallach@microsoft.com \\ Microsoft Research
}

\author{
Fernando Diaz* \\ diazf@acm.org \\ Spotify \\ Emine Yilmaz \\ emine.yilmaz@ucl.ac.uk \\ University College London
}

\begin{abstract}
Many online services, such as search engines, social media platforms, and digital marketplaces, are advertised as being available to any user, regardless of their age, gender, or other demographic factors. However, there are growing concerns that these services may systematically underserve some groups of users. In this paper, we present a framework for internally auditing such services for differences in user satisfaction across demographic groups, using search engines as a case study. We first explain the pitfalls of naïvely comparing the behavioral metrics that are commonly used to evaluate search engines. We then propose three methods for measuring latent differences in user satisfaction from observed differences in evaluation metrics. To develop these methods, we drew on ideas from the causal inference literature and the multilevel modeling literature. Our framework is broadly applicable to other online services, and provides general insight into interpreting their evaluation metrics.
\end{abstract}

\section{Keywords}

fairness; internal auditing methods; search engine evaluation; user demographics; user satisfaction

\section{INTRODUCTION}

Modern search engines are complex, relying heavily on machine learning methods to optimize search results for user satisfaction. Although machine learning can address many challenges in web search, there is also increasing evidence that suggests that these methods may systematically and inconspicuously underserve some groups of users 7,3 . From a social perspective, this is troubling. Search engines are a modern analog of libraries and should therefore provide equal access to information, irrespective of users' demographic factors 20]. Even beyond ethical arguments, there are practical reasons to provide equal access. From a business per-

*Work conducted while at Microsoft Research.

(C) 2017 International World Wide Web Conference Committee (IW3C2), published under Creative Commons CC BY 4.0 License. WWW 2017 Companion, April 3-7, 2017, Perth, Australia. ACM 978-1-4503-4914-7/17/04. http://dx.doi.org/10.1145/3041021.3054197

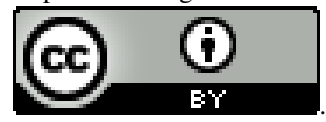

spective, equal access helps search engines attract a large and diverse population of users. From a public-relations perspective, service providers and the decisions made by their services are under increasing scrutiny by journalists 13 and civil-rights enforcement 38, 4] for seemingly unfair behavior.

One way to assess whether a search engine provides equal access is to look for differences in user satisfaction across demographic groups. If users from one group are consistently less satisfied than users from another, then these users are likely not being provided with equal search experiences. However, in practice, measuring differences in satisfaction is non-trivial. One demographic group may issue very different queries than another. Or, two groups may issue similar queries, but with different intents. Any differences in aggregate evaluation metrics will therefore reflect these contextual differences, as well as any differences in user satisfaction. Moreover, since user satisfaction cannot be measured at scale using explicit feedback, search engines often rely on implicit feedback based on behavioral signals, such as the number of clicks or the dwell time (i.e., the time spent on a page) 42. Even controlling for differences in the types of queries issued and in user intents, these signals may themselves be systematically influenced by demographics. Therefore, we cannot interpret evaluation metrics based on them as being direct reflections of user satisfaction. For example, if older users typically read more slowly than younger users, then a metric based on dwell time will, on average, be higher for older users, regardless of their levels of satisfaction.

To better understand the subtleties of this challenge, consider a search engine with users that span a wide range of age groups. As an example, suppose that users in their twenties comprise $80 \%$ of the search traffic, while users over the age of fifty comprise $10 \%$ of the search traffic. Suppose also that older users pose many more queries about retirement planning compared to younger users. Finally, suppose that the search engine relies on the dwell time for clicked results to measure user satisfaction 29]. It might seem natural to consider the average value of this metric in order to make product decisions. However, simply considering the average value of the metric across all users will underemphasize the effectiveness of the search engine on retirement planning queries. Moreover, if the search engine ranks documents poorly for retirement planning queries, then older users' low levels of satisfaction will be obscured. Even considering retirement planning queries in isolation, the average value may overemphasize the satisfaction of younger users focused on early-career retirement planning, again obscuring low levels 
of satisfaction for older users focused on late-career retirement planning. Finally, if the metric was calibrated with respect to younger users, then a dwell time of 30 seconds may be sufficient to demonstrate satisfaction. But if older users read more slowly, then a 30-second threshold might result in overoptimistic satisfaction estimates for these users.

In this paper, we propose three methods for measuring latent differences in user satisfaction from observed differences in evaluation metrics. All three methods are internal auditing methods - i.e., they use internal system information.

Our first two methods aim to disentangle user satisfaction from other demographic-specific variation by controlling for the effects of demographic factors on behavioral metrics; if we can recover an estimate of user satisfaction for each metric and demographic group pairing, then we can compare these estimates across groups. Any auditing method must strike a balance between generalizability and controlling for as many confounding factors as possible: the more controls in place, the less generalizable the conclusions. Our first method, context matching, controls for two confounding contextual differences: the query itself and the intent of the user (section 5). Because this method attempts to match users' search contexts as closely as possible, it can only be applied to a restricted set of queries. Our second method is a multilevel model for the effect of query difficulty on evaluation metrics (section 6). In contrast, this method controls for fewer confounding factors, but is more generalizable.

Although these methods shed light on observed differences between demographic groups, they say little about the substantive question of differential satisfaction across demographic groups. For our third method, we therefore take a different approach. Instead of estimating user satisfaction for each demographic group and then comparing these estimates, we estimate the latent differences directly (section (7). Because we are not interested in absolute levels of satisfaction, this is a more direct way to achieve our goal. This method infers which impression, among a randomly selected pair of impressions, led to greater user satisfaction. Then, using our second method, we set a threshold for differences that are so large that they are unlikely to explained by anything other than genuine differences in user satisfaction.

We used all three methods to audit Bing - a major search engine - using proprietary data focusing specifically on age and gender. We found significant differences in raw usage patterns and aggregate evaluation metrics for different demographic groups (section 4). However, after using our methods to control for confounding contextual differences, we found much less variation across groups (sections 5,6 , and 7). Overall, we found no difference in satisfaction between male and female users, but we did find that older users appear to be slightly more satisfied than younger users.

Finally, for comparison, we also used our third method to conduct an external audit of a leading competitor to Bing using publicly available data from comScore (section 8).

\section{RELATED WORK}

In this section, we briefly survey related work in three distinct research areas: fairness in machine learning, demographics and web search, and user satisfaction in web search.

\subsection{Fairness in Machine Learning}

As we described in the previous section, all three of our methods are internal auditing methods. Internal auditing methods are employed by service providers to review their own services using internal system information. For example, a social media platform might audit its own algorithms by examining the actual decisions made for the true population of users, with demographic attributes revealed. As another example, Feldman et al. presented a method for detecting and correcting potential demographic biases in training data 17. External auditing methods differ from internal auditing methods in that they rely only on publicly available information. External auditing methods are typically employed by third parties to review services without the cooperation of the service providers. As a result, external auditing methods cannot rely on internal system information. For example, Adler et al. presented a method for detecting biased decisions by probing an API [1]. Other work sought to detect unfairness through reverse A/B testing with synthetic users 31, 12, algorithmic auditing 37, and analysis of predictions made by supervised machine learning methods 24].

To the best of our knowledge, all previous work on internal and external auditing methods assumes that indicators of effectiveness are directly observable (e.g., accuracy). In contrast, our focus is on scenarios where the evaluation metrics themselves may be influenced by confounding demographic factors. Disentangling effectiveness from other demographic-specific variation is crucial in these scenarios.

\subsection{Demographics and Web Search}

Researchers have studied the behavior of search engine users in various settings: Ford et al. 18. conducted a controlled experiment involving masters students, varying age and gender; Weber and Castillo 40, 41] studied differences in user behavior using search log data; $\mathrm{Bi}$ et al. 6] demonstrated that search behavior can be used to predict demographic attributes; Lorigo et al. 32 studied the effect of gender on user behavior and found a relationship between gender and eye gaze patterns; and several studies 30, 35 have established that the search behavior of school-aged children varies by gender. Other related studies measured the impact of demographics on search results [23], examined the search engine manipulation effect 15, explored demographic context as a means to improve search results for ambiguous queries, and analyzed gender differences in search perceptions 45]. Together, these studies ground the role of demographics in evaluating search engines and motivate our work.

\subsection{User Satisfaction in Web Search}

Although search engines are often evaluated using metrics based on behavioral signals, several studies have suggested that these metrics are sensitive to a variety of factors: Hassan and White 26] demonstrated that evaluation metric values vary dramatically by user; Carterette et al. [10 made a similar observation and therefore incorporated user variability into evaluation metrics; and Borisov et al. studied the degree to which metrics are sensitive to a user's search context 8. Our work adopts a similar philosophy, focusing on measuring the extent to which demographics affect metrics.

\section{DATA AND METRICS}

We selected a random subset of Bing's desktop and laptop users from the English-speaking US market, and focused on their log data from a two week period during February, 2016. We removed spam using standard bot-filtering methods, and discarded queries that were not manually entered. By per- 
forming these filtering steps, we could be sure that any differences in evaluation metrics were not due to differences in devices, languages, countries, or query input methods.

We enriched these data with user demographics, focusing on self-reported age and (binary) gender information obtained during account registration. We discarded data from any users older than 74 , and binned the remaining users according to generational boundaries: (1) younger than 18 (post-millennial), (2) 18-34 (millennial), (3) 35-54 (generation X), and (4) 55-74 (baby boomers) ${ }^{1}$ To validate each user's self report, we predicted their age and gender from their search history, following the approach of $\mathrm{Bi}$ et al. 6. We then compared their predicted age and gender to their self-reported age and gender. If our prediction did not match their self report, we discarded their data. Approximately $51 \%$ of the remaining users were male. In contrast, the distribution of users across the four age groups was much less even, with the younger age groups containing substantially fewer users $(<1 \%$ and $13 \%$ for post-millennial and millennial, respectively) compared to the older age groups $(41 \%$ and $45 \%$ for generation $\mathrm{X}$ and baby boomers, respectively).

Finally, we labeled the remaining queries with topic information, using the approach of Bennett et al. 5]. For each query, we categorized the top three results, as well as all of the results clicked on by users, into the top two levels of the Open Directory Project ${ }^{2}$ topic hierarchy using a stateof-the-art text-based classifier. We then selected the most common topic from the categories predicted for that query.

After these steps, we were left with 32 million search impressions, involving 16 million distinct queries. (A search impression is a unique view of a results page presented to a user in response to a query.) These queries were issued by 4 million distinct users over 17 million distinct sessions.

Search engines log massive amounts of user interaction data that are retrospectively analyzed to detect, design, and validate behavioral signals that could serve as an implicit source of feedback. As described in the previous section, evaluation metrics based on these behavioral signals are often used as a proxy for user satisfaction. Drawing upon previous work, we considered four different evaluation metrics, each intended to operationalize user satisfaction: graded utility, reformulation rate, page click count, and successful click count. For graded utility, page click count, and successful click count, higher values mean higher user satisfaction; for reformulation rate, the relationship is reversed.

Graded utility is a model-based metric that provides a four-level estimate of user satisfaction based on search outcome and user effort. Jiang and Hassan 28] demonstrated experimentally that graded utility can predict subtle changes in satisfaction more accurately than other state-of-the-art methods, affording greater insight into search satisfaction.

Reformulation rate is the fraction of queries that were followed by another, reformulated, query. Reformulating a query is generally a strong indication that a user is dissatisfied with the search results for their original query. Hassan 25] showed that evaluation metrics based on reformulation rate successfully predict query-level satisfaction.

Page click count is the the total number of clicks made by a user on a results page. This evaluation metric is thought to reflect the user's level of engagement with the results page.

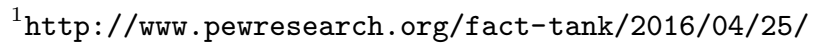
millennials-overtake-baby-boomers/

https://www.dmoz.org/
}

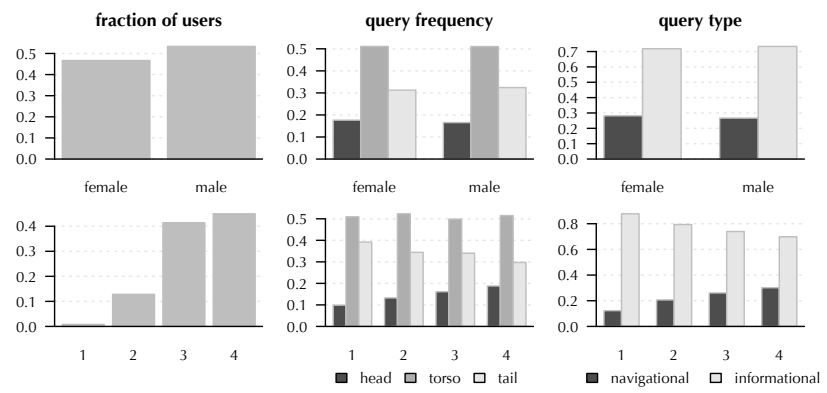

Figure 1: Raw usage patterns for queries issued by users with different genders (top) and age groups (bottom).

Although click-based evaluation metrics, such as page click count, have traditionally been used to measure user satisfaction, more engagement does not always correspond to higher satisfaction. Researchers have therefore proposed time-based metrics that are often more robustly correlated with user satisfaction. Successful click count is the number of clicked results with dwell times longer than 30 seconds 9 .

\section{DEMOGRAPHIC DIFFERENCES}

In this section, we describe observed differences between demographic groups. We focus on differences in the types of queries issued by users and differences in evaluation metrics.

\subsection{Differences in Queries}

First, we found that users from different demographic groups issued different types of queries (figure 1). As described in the previous section, roughly half of the users were male. However, a higher proportion of female users (28\%) issued navigational queries compared to male users (26\%). Although similar proportions of male and female users $(\sim 17 \%)$ issued head queries, slightly more male users issued tail queries. (The top $20 \%$ and bottom $30 \%$ of queries by search traffic are called head and tail queries, respectively.) Based on these differences alone, we would expect male users to exhibit worse values for the evaluation metrics described in the previous section. In contrast to gender, the distribution of users across the four age groups was much less even, with the younger age groups containing substantially fewer users than the older age groups. We found that a higher proportion of older users (30\%) issued navigational queries compared to younger users (13\%), while younger users (39\%) were more likely to issue tail queries compared to older users (30\%).

We also compared the actual queries issued by users from different demographic groups. Specifically, we computed the Kullback-Leibler divergence between pairs of (smoothed) distributions over queries issued by different age groups. The queries issued by the youngest age group were most similar to the second-youngest age group $\left(\mathrm{D}_{12}=0.0385\right)$ and least similar to the two oldest age groups $\left(\mathrm{D}_{13}=0.0415\right.$, $\left.\mathrm{D}_{14}=0.0480\right)$. We observed the same pattern for the other age groups, suggesting that users who are close in age are more likely to issue similar queries than users whose ages are further apart. Given the uneven distribution of users across age groups, we therefore hypothesize that evaluation metrics will be skewed to reflect topics queried by older users, potentially overlooking topics queried by younger users. 


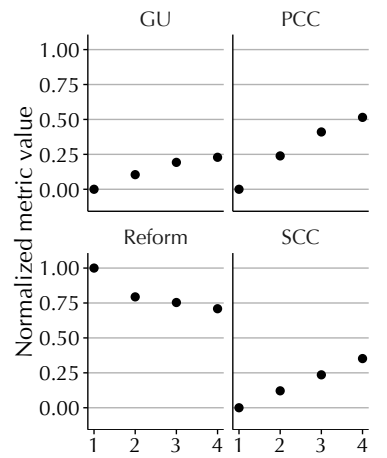

(a) age

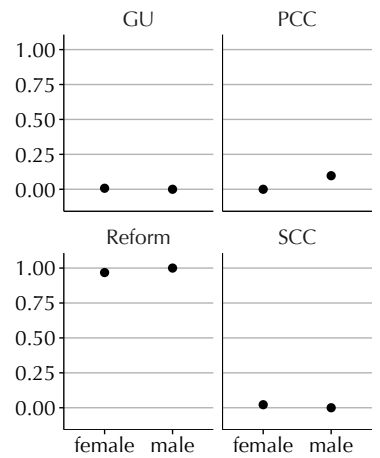

(b) gender
Figure 2: Raw normalized query-averaged values for each metric by age groups (a) and genders (b). "GU" denotes graded utility; "PCC" denotes page click count; "Reform" denotes reformulation rate; "SCC" denotes successful click count. Error bars (one standard error) are present in all plots, but are mostly so small that they cannot be seen.

\subsection{Differences in Evaluation Metrics}

Next, we compared the evaluation metrics described in section 3 across demographic groups, without controlling for any confounding demographic-specific variation. For each metric and demographic group pairing (e.g., graded utility and millennial), we computed the average metric value for each query issued by that group (by averaging over impressions) and then averaged these values. By computing query-averaged values, we ensured that our results were not dominated by the most popular queries. Finally, we normalized the query-averaged values to lie between zero and one. To do this, we identified the minimum and maximum values for each metric over the demographic groups, subtracted the corresponding minimum off of each value, and divided each result by the corresponding maximum minus the minimum.

We provide the normalized query-averaged values for each metric and age group pairing in figure 2a. The metrics all follow the same trend: older users have better values (lower for reformulation rate, higher for the other metrics) than younger users. Furthermore, the differences are quite large - for example, users in the youngest age group have a normalized query-averaged successful click count value of zero, while users in the oldest age group have a value of 0.31 . However, as described in section 1, we cannot conclude that this trend means that older users are genuinely more satisfied than younger users; these differences may be due to other demographic-specific variation. For example, users from different age groups issued different types of queries, so this trend may simply reflect this contextual difference.

In figure 2b, we provide the normalized query-averaged values for each metric and gender pairing. In contrast to age, there do not appear to be any differences between genders. Although this finding is reassuring, we cannot conclude that it means that male and female users are equally satisfied; there may be a large difference in satisfaction that is canceled out by other demographic-specific variation.

\section{CONTEXT MATCHING}

There are many possible sources of demographic-specific variation that could explain the results in the previous sec- tion, some of which may be difficult to observe and thus to control for. However, one obvious possibility is that the observed differences in evaluation metrics between demographic groups are due to differences in the types of queries issued. For example, if younger users issue harder queries than older users, then this could explain their lower values for the evaluation metrics. In this section, we present our first method for disentangling user satisfaction from other demographic-specific variation. This method recovers an estimate of user satisfaction for each metric and demographic group pairing by controlling for two confounding contextual differences: the query itself and the intent of the user.

We drew on well-established ideas from the causal inference literature to develop a matching method similar to those used in medicine and the social sciences 36]. Specifically, for each demographic factor (i.e., age or gender), we made sure that the impressions from that factor's groups were as close to identical as possible. By focusing on nearidentical contexts, we were able to control for as many sources of demographic-specific variation as possible. To do this, we used several filtering steps, each of which was selected to minimize the chance that any observed differences in evaluation metrics between demographic groups were due to anything other than genuine differences in user satisfaction.

We first restricted the data to navigational queries because they are generally less ambiguous than informational queries [39. We then retained only those queries with at least ten impressions from each demographic group. To control for the intent of the user, we followed the approach of Radlinski et al. 34. Specifically, for each query, we identified the search result with the most final successful clicks. (A final successful click is a successful click-i.e., a click with a dwell time longer than 30 seconds - that terminates the query.) We then discarded any impression whose final successful click was not on that result. Finally, to be certain that the users had the same choices available to them when making those clicks, we kept only those impressions with the same results page (up to the first eight results). After these steps, we were left with 1.2 million impressions, involving 19,000 distinct queries, issued by 617,000 distinct users.

Following the approach described in section 4 , for each metric and demographic group pairing, we computed the average metric value for each query issued by that group (by averaging over impressions) and then averaged these values. By considering only the 1.2 million impressions described in the previous paragraph, we could be sure we were comparing impressions that were for the same query with the same results page and which resulted in the same search result being the final successful click - a proxy for the intent of the user. That said, our filtering steps did not allow us to control for more subtle sources of demographic-specific variation.

In figure 3a, we provide normalized query-averaged values for each metric and age group pairing, computed using the context-matched data. There is much less variation across age groups than in figure $2 \mathrm{a}$ (all data). This finding suggests that the trend described in the previous section is unlikely to be due to genuine differences in user satisfaction. If the search engine were systematically underserving younger users, we would expect to see the same trend in figure $3 \mathrm{a}$ In figure $3 \mathrm{~b}$, we provide the normalized query-averaged values for each metric and gender pairing. As in figure $2 \mathrm{~b}$, there do not appear to be any differences between genders. The 


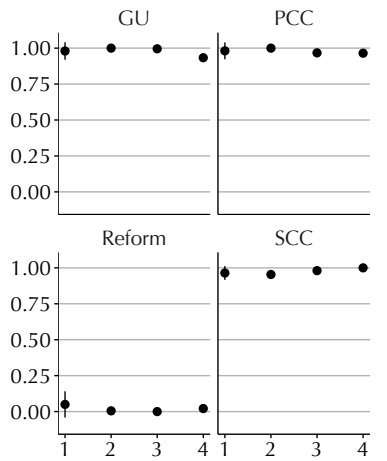

(a) age

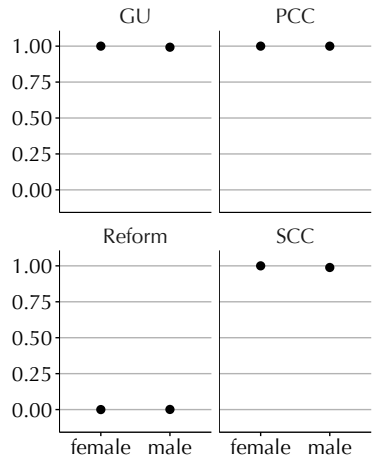

(b) gender
Figure 3: Context-matched normalized query-averaged values for each metric by age groups (a) and genders (b). "GU" denotes graded utility; "PCC" denotes page click count; "Reform" denotes reformulation rate; "SCC" denotes successful click count. Error bars (one standard error) are present in all plots, but are mostly so small that they cannot be seen.

similarity of these figures means that we can be reasonably confident that male and female users are equally satisfied 3

We note that the normalized query-averaged values computed using the context-matched data are significantly better (lower for reformulation rate, higher for the other metrics) than the values in section 4 . This is likely because our filtering steps restricted the data to queries that were popular enough to be issued ten or more times by each demographic group and that led to consistent result pages across impressions. Such queries are mostly head queries, which are generally associated with higher levels of satisfaction 14 .

As we described in section 1 any auditing method must strike a balance between generalizability and controlling for as many confounding factors as possible. Although our contextmatching method requires a restricted data set, making it less generalizable, it controls for both the query and the intent of the user, leading to a more reliable estimate of user satisfaction for each metric and demographic group pairing. Moreover, in many scenarios, focusing on only the most popular queries is a very reasonable thing to do, especially if these queries account for the majority of impressions.

\section{MULTILEVEL MODELING}

In this section, we present our second method for disentangling user satisfaction from other demographic-specific variation. Like our context-matching method, this method recovers an estimate of user satisfaction by controlling for confounding contextual differences; however, it only controls for characteristics of the query itself and not for the intent of the user. As a result, this method is more generalizable, and we were able to use it without restricting the data.

We drew on the multilevel modeling literature 22 to develop a new statistical model for the effect of query difficulty on evaluation metrics, controlling for the topic of the query

\footnotetext{
${ }^{3}$ We still cannot be completely sure because there still may be a large difference in satisfaction that is canceled out by other demographic-specific variation; however, because we considered impressions that were for the same query with the same results page and which resulted in the same search result being the final successful click, this is very unlikely.
}

and demographics of the user who issued the query. We then used this model to examine the effects of age and gender on each of the four evaluation metrics described in section 3 for fixed query difficulties and topics. Because the model does not control for the intent of the user, we cannot be sure that these effects are due to differences in user satisfaction.

The model operationalizes the following intuition: we expect that queries with different difficulties will lead to different metric values. We also expect that queries about different topics will lead to different metric values, as will queries issued by users with different demographics. The model uses two levels to capture this intuition: the first level accounts for differences across age, gender, and topic combinations, while the second level models the differences themselves.

Letting $Y_{i}$ denote the value of one of the four evaluation metrics described in section 3 (i.e., graded utility, reformulation rate, page click count, or successful click count) for the $i^{\text {th }}$ impression in our data set, the model assumes that

$$
\mathbb{E}\left[Y_{i}\right]=f^{-1}\left(\alpha_{a_{i} g_{i} t_{i}}+\beta_{a_{i} g_{i} t_{i}} X_{i}\right)
$$

where $f(\cdot)$ is a link function; $a_{i}$ and $g_{i}$ are the age and gender of the $i^{\text {th }}$ impression's user; and $t_{i}$ and $X_{i}$ are the topic and difficulty of the $i^{\text {th }}$ impression's query. We can interpret $\alpha_{a_{i} g_{i} t_{i}}$ and $\beta_{a_{i} g_{i} t_{i}}$ as the intercept and slope, respectively, of $Y_{i}$ with respect to $X_{i}$. The model has a different intercept and slope for each unique age, gender, and topic combination $a \times g \times t$. Therefore, if $a_{i}=a$, where $a \in\{1,2,3,4\}, g_{i}=g$, where $g \in\{$ male, female $\}$, and $t_{i}=t$, where $t$ is a unique topic, then $\alpha_{a_{i} g_{i} t_{i}}=\alpha_{a g t}$ and, similarly, $\beta_{a_{i} g_{i} t_{i}}=\beta_{a g t}$.

At the second level, the model further assumes that $\alpha_{a g t}$ and $\beta_{\text {agt }}$ are a linear combinations of age, gender, and topic indicator variables, as well as corresponding interaction terms:

$$
\left(\begin{array}{c}
\alpha_{a g t} \\
\beta_{a g t}
\end{array}\right)=\left(\begin{array}{c}
\mu_{0} \\
\mu_{1}
\end{array}\right)+\left(\begin{array}{c}
\alpha_{a} \\
\beta_{a}
\end{array}\right)+\left(\begin{array}{c}
\alpha_{g} \\
\beta_{g}
\end{array}\right)+\left(\begin{array}{c}
\alpha_{t} \\
\beta_{t}
\end{array}\right)+\left(\begin{array}{c}
\alpha_{a \times g \times t} \\
\beta_{a \times g \times t}
\end{array}\right) .
$$

Finally, the model assumes that the coefficients at the second level are drawn from a mean-zero Gaussian distribution:

$$
\left(\begin{array}{l}
\alpha_{k} \\
\beta_{k}
\end{array}\right) \sim \mathcal{N}\left(\left(\begin{array}{l}
0 \\
0
\end{array}\right), \Sigma_{k}\right) \text { where } k \in\{a, g, t\} .
$$

To estimate the difficulty of each query, we sorted the queries issued by each demographic group according to their graded utility values. We then averaged each query's percentile positions in these lists to obtain an estimate of its difficulty that is uncorrelated with the demographics of the users who issued it. Most methods for estimating the difficulty of a query are based on behavioral signals, such as the reformulation rate or the dwell time 16, 43. Because behavioral signals may themselves be systematically influenced by demographics, we were unable to use these methods.

We used a random sample of 1.4 million impressions to fit a different version of the model for each evaluation metric. Because graded utility ranges from negative one to positive one, we used a Gaussian model with an identity link function; because reformulation rate ranges from zero to one, we used a binomial model with a logit link function; and, because page click count and successful click count are both non-negative integers, we used a Poisson model with a logarithmic link function. We fit each evaluation metric's version of the model using Bayesian inference techniques 21].

Overall, we found varying levels of satisfaction across different topics. We also found that satisfaction decreased with query difficulty. Again, we found that gender had little ef- 
fect on any of the metrics, while age had an effect on all four. For each topic and age group pairing, we used each metric's version of the model (with $g_{i}$ arbitrarily fixed to male) to predict the values of that metric for query difficulties between zero and one in increments of 0.05. In figures $4 \mathrm{a}$ and $4 \mathrm{~b}$ we depict these values for graded utility and page click count; we show only the six hardest query difficulties. These plots indicate that older users have slightly higher values than younger users. In figures $4 \mathrm{c}$ and $4 \mathrm{~d}$ we depict these values for successful click count; we show the six easiest and six hardest query difficulties. Again, older users have slightly higher values than younger users. This difference is more pronounced for more difficult queries, suggesting that age has a bigger effect on satisfaction for these queries.

Although we found that age had an effect on all four satisfaction metrics, we cannot conclude that our results mean that older users are more satisfied than younger users. Because our model only controls for the topic of the query and the demographics of the user who issued the query, these differences may be due to differences in intent or other demographic-specific variation, as well as differences in user satisfaction. Irrespective of the cause of these differences, the contrast between these results and the results in the previous section highlights the need for evaluation metrics that are not confounded by demographic-specific variation.

\section{ESTIMATING DIFFERENCES}

Although the methods described in sections 5 and 6 shed light on observed differences in evaluation metrics between demographic groups, they do not directly address our goal of measuring latent differences in user satisfaction. For our third method, which we present in this section, we therefore take a different approach. Rather than estimating absolute levels of satisfaction for each demographic group and then comparing these estimates, this method estimates differences in satisfaction between demographic groups directly. First, it considers randomly selected pairs of impressions (for the same query, issued by users from different demographic groups) and uses a high-precision algorithm to estimate which impression led to greater user satisfaction. Using these labels, it then models differences in satisfaction.

We restricted the data to only those queries that were issued by users from at least three demographic groups and that had at least ten impressions. We then randomly selected $10 \%$ (roughly 62,000 ) of these queries. For each query, we randomly selected 10,000 pairs of impressions, resulting in a total of 2.7 billion pairs. Finally, for each pair, we compared the impressions' values of the evaluation metrics and labeled one of the impressions as leading to greater user satisfaction if there was a difference so large that it was unlikely to be explained by anything other than a genuine difference in user satisfaction. By performing these preprocessing and labeling steps, we were able to construct a high-precisionlow-recall proxy for pairwise differences in user satisfaction.

We provide the algorithm that we used to compare the impressions' metric values in figure $5 \mathrm{a}$ The metrics are ordered according to importance. For example, reformulation rate is thought to be a strong indicator of dissatisfaction. The algorithm therefore considers reformulation rate first. If there is a difference, it returns a label without considering the other metrics. If there is no difference, it moves on to consider graded utility, followed by successful click count. Finally, it considers graded utility and successful click count together, using a slightly less conservative threshold; if there are differences in both metrics, these differences are more likely to reflect genuine differences in user satisfaction.

We obtained the thresholds using the model described in the previous section. Specifically, we used our estimates of the effects of demographics factors on the metrics to derive conservative upper bounds on the effects of demographicspecific variation. For each metric, we then used the corresponding bound to derive a minimum threshold for differences that are so large that they are unlikely to be explained by anything other than genuine differences in user satisfaction. For example, if the difference in graded utility between groups had a maximum of $\delta$, then we set the minimum threshold to $k \delta$, where $k>1$ reflects our confidence that two impressions whose graded utility values differ by at least $k \delta$ correspond to a genuine difference in user satisfaction. A higher value of $k$ yields a higher-precision-lower-recall algorithm. We set $k=2.5$ to obtain the values in figure $5 \mathrm{a}$.

We used a single-level model to estimate latent differences in user satisfaction across demographic groups. This model is similar to the one described in the previous section, but does not include query-specific terms because we restricted the data to pairs of impressions for the same query. Letting $S_{i}-S_{j}$ denote the latent difference in user satisfaction between the $i^{\text {th }}$ and $j^{\text {th }}$ impressions, the model assumes that

$$
\begin{aligned}
& P\left(S_{i}-S_{j}>0\right)= \\
& \quad f^{-1}\left(\mu_{0}+\gamma_{a_{i}}+\gamma_{a_{j}}+\gamma_{g_{i}}+\gamma_{g_{j}}+\gamma_{a_{i} \times g_{i} \times a_{j} \times g_{j}}\right),
\end{aligned}
$$

where $f(\cdot)$ is a logit link function and $a_{i} \times g_{i} \times a_{j} \times g_{j}$ denotes an interaction term. The model also assumes that the coefficients are Gaussian distributed around zero.

We fit the model using pairs of impressions from different demographic groups, labeled as either +1 or -1 via the algorithm in figure 5a. Again, we found that gender had little effect. To compare differences in satisfaction across age groups, we arbitrarily fixed $g_{i}$ and $g_{j}$ to male and female, respectively. Then, for each age group pairing, we used the model to predict $P\left(S_{i}-S_{j}>0\right)$. We visualize the probabilities for each pairing in figure $6 a$ This figure suggests that older users are more satisfied than younger users, with larger differences for users whose ages are further apart; however, because the probabilities are all close to 0.5 , the difference is relatively small for each age group pairing. These findings are consistent with the findings described in sections 4 and 6 though, again, we note that these differences may be due to other unmodeled demographic-specific variation.

\section{EXTERNAL AUDITING}

To demonstrate the generality of our third method, we used this method to conduct an external audit of a leading competitor to Bing. We used publicly available data provided by comScore, an Internet analytics company 4 To assemble this data set, comScore recruited an opt-in consumer panel, validated to be representative of the online population and projectable to the total US population [19]. The data set consists of unfiltered search queries collected over a one week period during November 2011. Due to a lack of detailed behavioral signals, we used only page click count to operationalize user satisfaction. We followed the approach described in section 7, again focusing on age and gender,

${ }_{4}^{4}$ htp://www.comscore.com/Products/ Audience-Analytics/qSearch 


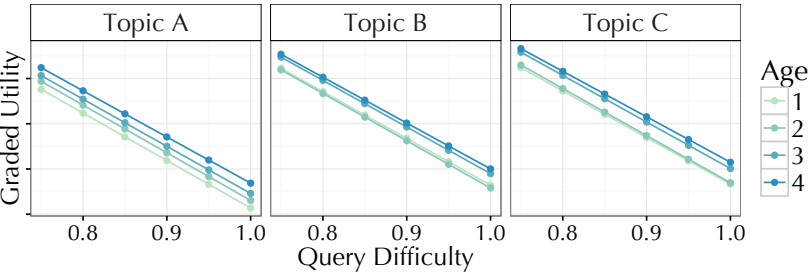

(a) graded utility (hardest queries)

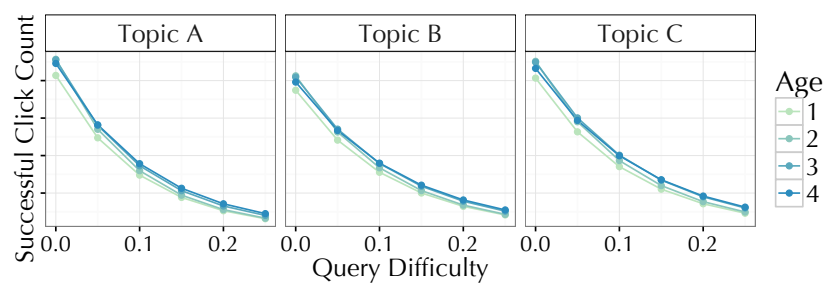

(c) successful click count (easiest queries)

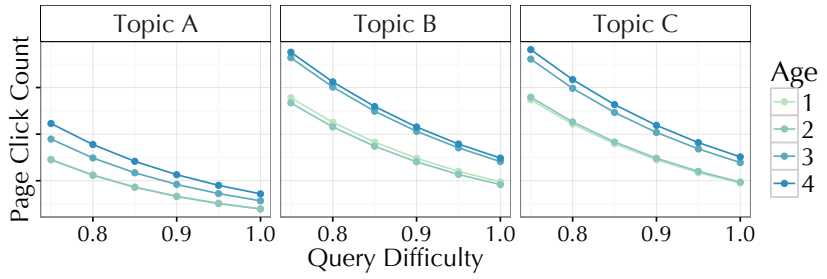

(b) page click count (hardest queries)

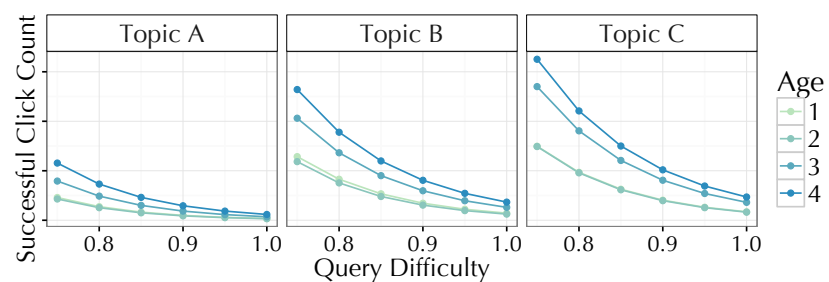

(d) successful click count (hardest queries)

Figure 4: Evaluation metrics according to our model. For graded utility (a) and page click count (b), we show only the six hardest query difficulties; for successful click count, we show the six easiest (c) and six hardest (d) query difficulties.

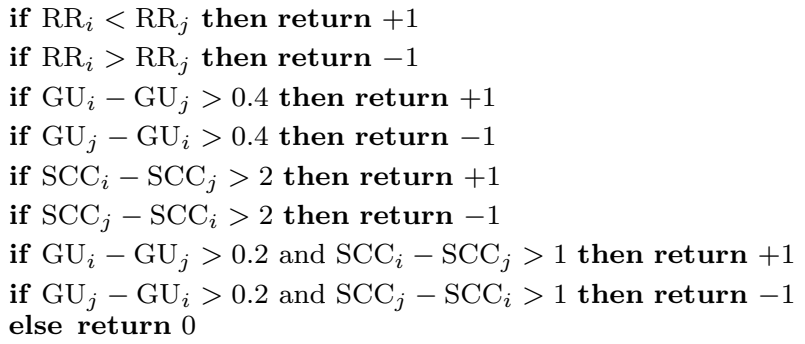

(a) Bing

if $\mathrm{PCC}_{i}-\mathrm{PCC}_{j}>2$ then return +1 if $\mathrm{PCC}_{j}-\mathrm{PCC}_{i}>2$ then return -1 else return 0

(b) comScore

Figure 5: Algorithms for labeling a pair of impressions.

but rather than using the algorithm in figure $5 \mathrm{a}$, we labeled each pair of impressions using the algorithm in figure $5 \mathrm{~b}$

We fit the model described in the previous section (i.e., equation 4h using 1.2 million pairs of impressions from different demographic groups, labeled as either +1 or -1 via the algorithm in figure $5 \mathrm{~b}$ Again, we found that gender had little effect, so we arbitrarily fixed $g_{i}$ and $g_{j}$ to male and female, respectively. For each age group pairing, we then used the model to predict $P\left(S_{i}-S_{j}>0\right)$. We visualize the probabilities for each pairing in figure $6 \mathrm{~b}$ Similar to the results in the previous section, this figure suggests that older users tend to be slightly more satisfied than younger users.

\section{DISCUSSION}

Internally auditing search engines for equal access is much more complicated than comparing evaluation metrics for demographically binned search impressions. In this paper, we addressed this challenge by proposing three methods for measuring latent differences in user satisfaction from observed differences in evaluation metrics. We then used these methods to audit Bing, focusing specifically on age and gen-

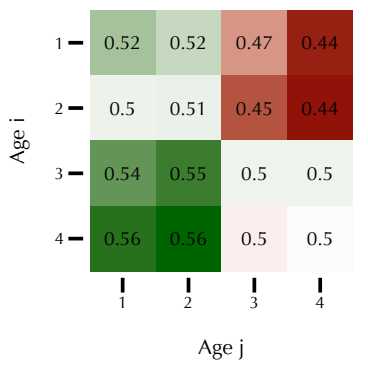

(a) Bing

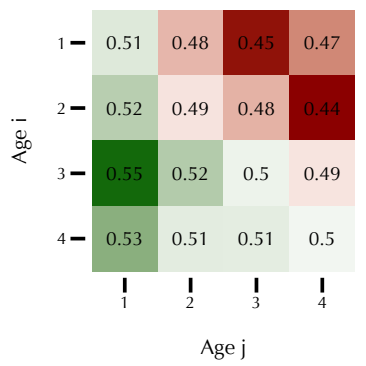

(b) comScore
Figure 6: $P\left(S_{i}-S_{j}>0\right)$ for each age group pairing. Standard errors (not shown) are between 0.001 and 0.004 .

der. Overall, we found no difference in satisfaction between male and female users, but we did find that older users appear to be slightly more satisfied than younger users. Because we used three different methods, with complementary strengths, we can be confident that any trends detected by all three methods are genuine, though we cannot conclude that they were due to differences in user satisfaction, as opposed to unmodeled demographic-specific variation.

We then used our third method to conduct an external audit of a leading competitor to Bing, using publicly available data from comScore. Again, we found that older users tend to be slightly more satisfied than younger users. Because we saw the same trends for two independently developed search engines, we hypothesize that these trends are likely due to unmodeled differences between demographic groups, rather than genuine differences in user satisfaction. That said, we believe that this finding is important and should be explored further. Graded utility and successful click count both depend on dwell-time thresholds. Although previous laboratory experiments have not shown substantial differences in reading times between older and younger populations 2, other work has shown differences in reading times for scenarios involving a mixture of relevant and non-relevant text 11 . 
Moreover, many online services other than search engines also use evaluation metrics based on dwell time 27, 33, 44].

We conclude that there is a need for further investigation into observed differences in evaluation metrics across demographic groups, as well as a need for new metrics that are not confounded with demographics and can be computed without using costly explicit feedback elicitation methods.

\section{REFERENCES}

[1] P. Adler, C. Falk, S. A. Friedler, G. Rybeck, C. Scheidegger, B. Smith, and S. Venkatasubramanian. Auditing black-box models for indirect influence. arXiv:1602.07043.

[2] H. Ajutsu, G. E. Legge, J. A. Ross, and K. J. Scheubel. Psychophysics of reading-X. Effects of age-related changes in vision. Journal of Gerontology, 46(6):325-331, 1991.

[3] J. Angwin, J. Larson, S. Mattu, and L. Kirchner. Machine bias. https://www.propublica.org/article/ machine-bias-risk-assessments-in-criminal-sentencing 2016.

[4] S. Barocas and A. D. Selbst. Big data's disparate impact. California Law Review, 104:671-732, 2016.

[5] P. N. Bennett, K. Svore, and S. T. Dumais. Classification-enhanced ranking. In $W W W, 2010$

[6] B. Bin, M. Shokouhi, M. Kosinski, and T. Graepel. Inferring the demographics of search users: Social data meets search queries. In $W W W, 2013$.

[7] T. Bolukbasi, K.-W. Chang, J. Zou, V. Saligrama, and A. Kalai. Man is to computer programmer as woman is to homemaker? Debiasing word embeddings. In NIPS, 2016.

[8] A. Borisov, I. Markov, M. de Rijke, and P. Serdyukov. A context-aware time model for web search. In SIGIR, 2016

[9] G. Buscher, L. van Elst, and A. Dengel. Segment-level time as implicit feedback: A comparison to eye tracking. In SIGIR, 2009.

[10] B. Carterette, E. Kanoulas, and E. Yilmaz. Incorporating variability in user behavior into systems based evaluation. In CIKM, 2012.

[11] S. L. Connelly, L. Hasher, and R. T. Zacks. Age and reading: The impact of distraction. Psychology and Aging, 6(4):533-541, 1991.

[12] A. Datta, S. Sen, and Y. Zick. Algorithmic transparency via quantitative input influence. In Proceedings of the Thirty-Seventh IEEE Symposium on Security and Privacy, 2016.

[13] N. Diakopoulos. Algorithmic accountability. Digital Journalism, 3(3):398-415, 2015

[14] D. Downey, S. Dumais, and E. Horvitz. Heads and tails: Studies of web search with common and rare queries. In SIGIR, 2007.

[15] R. Epstein and R. E. Robertson. The search engine manipulation effect (SEME) and its possible impact on the outcomes of elections. PNAS, 2015.

[16] H. A. Feild, J. Allan, and R. Jones. Predicting searcher frustration. In SIGIR, 2010.

[17] M. Feldman, S. A. Friedler, J. Moeller, C. Scheidegger, and S. Venkatasubramanian. Certifying and removing disparate impact. In $K D D, 2015$.

[18] N. Ford, D. Miller, and N. Moss. Web search strategies and human individual differences: Cognitive and demographic factors, internet attitudes, and approaches. JASIST, 56(7):741-756, 2005.

[19] G. M. Fulgoni. The "professional respondent" problem in online survey panels today. Presented at the Marketing Research Association Annual Conference, 2005.

[20] L. Garcia-Febo, A. Hustad, H. Rösch, P. Sturges, and A. Vallotton. IFLA code of ethics for librarians and other information workers. International Federation of Library Associations and Institutions, 2012.

[21] A. Gelman, J. B. Carlin, H. S. Stern, D. B. Dunson, A. Vehtari, and D. B. Rubin. Bayesian Data Analysis. CRC Press, 2014.

[22] A. Gelman and J. Hill. Data Analysis Using Regression and Multilevel/Hierarchical Models. Cambridge University Press, 2006.

[23] A. Hannak, P. Sapiezynski, A. M. Kakhki, B. Krishnamurthy, D. Lazer, A. Mislove, and C. Wilson. Measuring personalization of web search. In $W W W, 2013$.

[24] M. Hardt, E. Price, and N. Srebro. Equality of opportunity in supervised learning. In NIPS, 2016
[25] A. Hassan, X. Shi, N. Craswell, and B. Ramsey. Beyond clicks: Query reformulation as a predictor of search satisfaction. In CIKM, 2013.

[26] A. Hassan and R. W. White. Personalized models of search satisfaction. In $C K I M, 2013$.

[27] Y. Hu, Y. Koren, and C. Volinsky. Collaborative filtering for implicit feedback datasets. In ICDM, 2008.

[28] J. Jiang, A. Hassan, Z. Shi, and R. W. White. Understanding and predicting graded search satisfaction. In WSDM, 2015.

[29] Y. Kim, A. Hassan, R. W. White, and I. Zitouni. Modeling dwell time to predict click-level satisfaction. In WSDM, 2014

[30] A. Large, J. Beheshti, and T. Rahman. Gender differences in collaborative web searching behavior: An elementary school study. Information Processing and Management, 2002.

[31] M. Lecuyer, R. Spahn, Y. Spiliopoulos, A. Chaintreau, R. Geambasu, and D. Hsu. Sunlight: Fine-grained targeting detection at scale with statistical confidence. In CCS, 2015.

[32] L. Lorigo, B. Pan, H. Hembrooke, T. Joachims, L. Granka, and G. Gay. The influence of task and gender on search and evaluation behavior using Google. Information Processing and Management, 2006.

[33] E. R. Núñez-Valdéz, J. M. C. Lovelle, O. S. Martínez, V. García-Díaz, P. O. de Pablos, and C. Marín. Implicit feedback techniques on recommender systems applied to electronic books. Computers in Human Behavior, 28(4):1186-1193, 2012.

[34] F. Radlinski, M. Szummer, and N. Craswell. Inferring query intent from reformulations and clicks. In $W W W, 2010$.

[35] M. Roy and M. T. H. Chi. Gender differences in patterns of searching the web. Journal of Educational Computing Research, 29(3), 2003.

[36] D. B. Rubin. Matched Sampling for Causal Effects. Cambridge University Press, 2006.

[37] C. Sandvig, K. Hamilton, K. Karahalios, and C. Langbort. Auditing algorithms: Research methods for detecting discrimination on internet platforms. Presented at Data and Discrimination: Converting Critical Concerns into Productive Inquiry: A Preconference to the Sixty-Fourth Annual Meeting of the International Communication Association, 2014.

[38] M. Smith, D. Patil, and C. Muñoz. Big data: A report on algorithmic systems, opportunity, and civil rights. Technical report, Executive Office of the President of the United States, 2016

[39] Y. Wang and E. Agichtein. Query ambiguity revisited: Clickthrough measures for distinguishing informational and ambiguous queries. In $N A A C L, 2010$.

[40] I. Weber and C. Castillo. The demographics of web search. In SIGIR, 2010.

[41] I. Weber and A. Jaimes. Who uses web search for what: and how. In WSDM, 2011.

[42] R. W. White. Interactions with Search Systems. Cambridge University Press, 2016.

[43] R. W. White and S. Dumais. Characterizing and predicting search engine switching behavior. In $C I K M, 2009$

[44] P. Yin, P. Luo, W.-C. Lee, and M. Wang. Silence is also evidence: Interpreting dwell time for recommendation from psychological perspective. In $K D D, 2013$.

[45] M. Zhou. Gender difference in web search perceptions and behavior: Does it vary by task performance? Computers and Education, 78:174-184, 2014. 